\title{
Toxicidad sobre piel y mucosas: tratamientos de soporte
}

\author{
A. Montero Luis, A. Hervás, R. Morera, A. Ramos
}

\section{Introducción}

La aparición de mucositis durante el tratamiento con radioterapia y/o radioquimioterapia representa la toxicidad no hematológica más frecuente. Se asocia con una morbilidad importante: dolor, odinofagia, hipogeusia, deshidratación e incluso malnutrición. La aparición de alteraciones en la piel durante la radioterapia es una consecuencia inevitable, hasta cierto punto, de la misma. La utilización cada vez más extendida de aceleradores lineales para los tratamientos y de técnicas para proteger la piel ha disminuido de forma notable la intensidad y severidad de estas reacciones. No obstante, el empleo creciente de esquemas de tratamiento que combinan radio y quimioterapia de forma simultánea así como las dosis progresivamente mayores de radioterapia hacen que la toxicidad cutánea continúe siendo un problema de primera magnitud en la práctica clínica diaria.

Todos los pacientes sometidos a radioterapia y/o radioquimioterapia sobre áreas de cabeza y cuello desarrollarán mucositis oral en algún momento del tratamiento, si bien la severidad de la misma dependerá tanto de las características del paciente como de las del tratamiento aplicado. Se estima que aproximadamente el $60 \%$ de los pacientes que reciben radioterapia exclusiva y más del $90 \%$ de aquellos en tratamiento con esquemas combinados de radio y quimioterapia desarrollarán un cuadro de mucositis severa ${ }^{1}$. En un porcentaje no despreciable de ellos, esta complicación obligará la suspensión temporal o definitiva del tratamiento siendo incluso necesario en ocasiones el ingreso hospitalario para el control de los síntomas y asegurar el aporte nutricional.

La incidencia real de epitelitis aguda es difícil de determinar. Se estima que entre el $80 \%$ y $90 \%$ de todos los pacien-

Servicio de Oncología Radioterápica

Hospital Ramón y Cajal

Madrid tes sometidos a radioterapia experimentan algún grado de afectación cutánea, principalmente eritema, y que un 10$15 \%$ sufren grados más avanzados como descamación húmeda o ulceración de la piel ${ }^{2}$.

Un reciente estudio acerca de los efectos de la irradiación en mujeres con cáncer de mama, observó que más del $90 \%$ de las pacientes presentaban algún grado de toxicidad durante el tratamiento, pero que la incidencia de toxicidad limitante (grados 3-4) era inferior al $10 \%^{3}$.

La aparición y severidad de la toxicidad sobre piel y mucosas estará en estrecha relación con una serie de factores dependientes tanto del propio paciente, como de las características del tratamiento realizado.

Factores dependientes del paciente y de su estado:

- Edad.

- Estado nutricional.

- Hábitos tóxicos asociados, en especial consumo de alcohol y tabaco.

- Presencia de comorbilidades: anemia, diabetes, esclerodermia, infección por virus de la inmunodeficiencia humana.

\section{Factores relacionados con el tratamiento:}

- Dosis total, volumen de tratamiento, energía, fraccionamiento.

- Utilización de quimioterapia concomitante.

- Cirugía previa.

\section{Evaluación de la toxicidad}

Las escalas más frecuentemente utilizadas para evaluar el grado e intensidad de la mucositis y epitelitis durante y después de la irradiación son las establecidas por el Radiation Therapy Oncology Group/European Organization for Research and Treatment of Cancer (RTOG/EORTC) ${ }^{4}$ o por el National Cancer Institute (NCI.CTC versión 3.0). Ambas establecen la existencia de 5 grados en función de la intensidad de la afectación, desde la no presencia de cambios con respecto a la situación basal (Grado 0) hasta la aparición de ulceración y/o necrosis (Grado 4). (Figs. 1 y 2). 


\section{MUCOSITIS AGUDA:}

RTOG/EORTC Criterios de toxicidad

\begin{tabular}{llll}
\hline 0 & 1 & 2 & 3 \\
\hline Sin cambios & $\begin{array}{l}\text { Eritema, dolor leve que } \\
\text { no requiere analgesia }\end{array}$ & $\begin{array}{l}\text { Mucositis parcheada, } \\
\text { dolor moderado que } \\
\text { precisa analgesia }\end{array}$ & $\begin{array}{l}\text { Mucositis confluente, } \\
\text { dolor severo }\end{array}$ \\
\hline
\end{tabular}

RTOG, Radiation Therapy Oncology Group; EORTC, European Organization for Research and Treatment of Cancer

NCI COMMON TOXICITY CRITERIA (CTC) Version 3.0

\begin{tabular}{llll}
\hline 0 & 1 & 2 & 3 \\
\hline Sin cambios & eritema & $\begin{array}{l}\text { Mucositis parcheada } \\
\text { pseudomembranosa } \\
\text { (parches } \leq 1.5 \mathrm{~cm} \text { y no } \\
\text { confluentes) }\end{array}$ & $\begin{array}{l}\text { Mucositis confluente } \\
\text { pseudomembranosa } \\
\text { (parches confluentes } \\
>1.5 \mathrm{~cm})\end{array}$ \\
\hline
\end{tabular}

\section{EPITELITIS AGUDA:}

\section{RTOG/EORTC Criterios de Toxicidad}

\begin{tabular}{llll}
\hline 0 & 1 & 2 & 3 \\
\hline Sin cambios & $\begin{array}{l}\text { Eritema, depilación, } \\
\text { descamación seca }\end{array}$ & $\begin{array}{l}\text { Eritema brillante, } \\
\text { descamación húmeda } \\
\text { parcheada, edema moderado }\end{array}$ & $\begin{array}{l}\text { Descamación } \\
\text { húmeda confluente, } \\
\text { edemportante }\end{array}$ \\
\hline
\end{tabular}

RTOG, Radiation Therapy Oncology Group; EORTC, European Organization for Research and Treatment of Cancer

NCI COMMON TOXICITY CRITERIA (CTC) Version 3.0

\begin{tabular}{llll}
\hline 0 & 1 & 2 & 3 \\
\hline Sin cambios & $\begin{array}{l}\text { Eritema, descamación } \\
\text { seca }\end{array}$ & $\begin{array}{l}\text { Eritema, descamación } \\
\text { húmeda parcheada } \\
\text { edema moderado }\end{array}$ & $\begin{array}{l}\text { Descamación húmeda } \\
\text { confluente }>1,5 \mathrm{~cm}\end{array}$ \\
\hline
\end{tabular}

Fig. 1.

\section{Tratamiento}

\section{Medidas generales}

Es importante informar y concienciar al paciente acerca de la necesidad de mantener una adecuada higiene de la piel y las mucosas antes, durante y después del tratamiento ${ }^{5-7}$.

Hay que realizar un meticuloso examen de la cavidad bucal antes de la irradiación, eliminando focos de posible infección y procediendo a la extracción de las piezas dentarias en mal estado. Igualmente es conveniente hacer saber al paciente que debe abstenerse de utilizar prótesis dentarias durante el tratamiento excepto en el momento de las comidas, así como evitar aquellos factores que contribuyan a la irritación de la mucosa como comidas excesivamente calientes, picantes o especiadas, bebidas alcohólicas, tabaco...

Igualmente es indispensable prevenir la desnutrición me- diante el consejo dietético y el aporte de los suplementos nutricionales necesarios.

Especialmente importantes son las recomendaciones que se deben hacer a todos los pacientes a fin de mantener una adecuada higiene de la piel. Debe evitarse la utilización de perfumes, colonias y desodorantes así como el empleo de antisépticos que contengan agua oxigenada, alcohol, yodo o mercurio y sus derivados. El afeitado se llevará a cabo preferiblemente con maquinilla eléctrica y no con cuchillas. Será recomendable vestir con ropas sueltas preferiblemente de algodón y proteger la piel de la exposición directa al sol, viento $y$ temperaturas extremas.

Hay que emplear analgésicos potentes desde las primeras manifestaciones de dolor con el fin de impedir que empeore el estado general así como el estado nutricional por la dificultad añadida que la mucositis supone para la ingesta oral. En muchos casos es preciso recurrir a la utilización de opiáceos siendo preferible emplear aquellos que se administran por vía 


\section{A. Montero Luis y cols.}

TOXICIDAD TARDÍA

\section{TOXOCIDAD MUCOSA Y GLANDULAR:}

RTOG/EORTC Criterios de toxicidad

\begin{tabular}{llll}
\hline 0 & 1 & 2 & 3 \\
\hline Sin cambios & $\begin{array}{l}\text { Sequedad o atrofia } \\
\text { leve, buena respuesta a } \\
\text { estímulos }\end{array}$ & $\begin{array}{l}\text { Sequedad o atrofia } \\
\text { moderada, pobre } \\
\text { respuesta a estímulos }\end{array}$ & $\begin{array}{l}\text { Atrofia severa, } \\
\text { sequedad completa, } \\
\text { no respuesta a estímulos }\end{array}$ \\
\hline
\end{tabular}

RTOG, Radiation Therapy Oncology Group; EORTC, European Organization for Research and Treatment of Cancer

\section{TOXICIDAD PIEL Y TEJIDO SUBCUTÁNEO:}

RTOG/EORTC Criterios de Toxicidad

\begin{tabular}{|c|c|c|c|c|}
\hline 0 & 1 & 2 & 3 & 4 \\
\hline Sin cambios & $\begin{array}{l}\text { Atrofia leve, fibrosis } \\
\text { leve, } \\
\text { hiperpigmentación, } \\
\text { pérdida de grasa } \\
\text { subcutánea }\end{array}$ & $\begin{array}{l}\text { Atrofia parcheada, } \\
\text { telangiectasias } \\
\text { moderadas, fibrosis } \\
\text { moderada, contractura } \\
\text { leve }\end{array}$ & $\begin{array}{l}\text { Atrofia severa, } \\
\text { telangiectasias } \\
\text { marcadas; induración } \\
\text { severa, pérdida del } \\
\text { tejido celular } \\
\text { subcutáneo, } \\
\text { contractura severa }\end{array}$ & Ulceración, necrosis \\
\hline
\end{tabular}

TOG, Radiation Therapy Oncology Group; EORTC, European Organization for Research and Treatment of Cancer

Fig. 2.

transdérmica (fentanilo, buprenorfina) que evitan la molestia asociada a la ingesta oral. En los episodios de dolor irruptivo pueden emplearse también por vía tópica aplicándolo directamente sobre la mucosa oral (citrato de fentanilo).

\section{Tratamiento específico}

\section{Antisépticos. Antibióticos. Antifúngicos:}

Debido a los fenómenos de pérdida de la integridad de la barrera epitelial y mucosa, los cambios en el flujo y composición de la saliva y la leucopenia asociada a la quimioterapia, la mucosa de la cavidad oral de los pacientes sometidos a radio y/o radioquimioterapia puede verse colonizada por un gran número de microorganismos potencialmente patógenos, especialmente gram positivos, gram negativos y hongos.

Por este motivo se ha postulado la utilización de antisépticos y antimicóticos tanto para la prevención como el tratamiento de la toxicidad aguda sobre piel y mucosas ${ }^{8-11}$.

Las infecciones asociadas ya establecidas deben combatirse enérgicamente con antibióticos y/o antifúngicos. Muy pocos agentes han demostrado realmente su utilidad en la prevención. No obstante, parece recomendable aconsejar sistemáticamente enjuagues con antisépticos no irritantes (povidona iodada) y ocasionalmente con antibióticos o antifúngicos.

\section{Protectores de la barrera epitelial y mucosa}

Antiinflamatorios

La manzanilla es una sustancia de conocidas actividades antiinflamatorias, antipépticas, antibacterianas y espasmolíticas. Se utiliza habitualmente en forma de lavados y enjuagues locales para la prevención y/o tratamiento de la toxicidad leve ${ }^{12}$.

Los emolientes como el ácido pantoténico, la urea, el aloe vera, la avena, proporcionan alivio sintomático y mejoran la calidad de vida de los pacientes al evitar la desecación de la capa córnea de la piel por efecto oclusivo, pero no aportan hidratación a la piel. Es conveniente utilizarlos desde el primer día de la irradiación ${ }^{13-19}$.

El sucralfato es una sal de aluminio no absorbible utilizada en el tratamiento de las úlceras gástricas. Actúa mediante la unión a proteínas de la mucosa formando una barrera mecánica. También posee un una acción cicatrizante mediante la estimulación del crecimiento celular al inducir la síntesis de prostaglandinas y del factor de crecimiento epitelial (EGF) así como incrementando la circulación epitelial y la producción de moco. Por último que actúa como antiinflamatorio local y tiene acción antibacteriana propia ${ }^{20-23}$.

Otra sal de aluminio hidratada utilizada con cierta frecuencia es el caolin-pectina, empleada habitualmente para el tratamiento de cuadros diarreicos. Su acción se basa en la formación de una barrera mecánica sobre la mucosa daña$\mathrm{da}^{24}$.

La benzidamina, un antiinflamatorio no esteroideo con propiedades antisépticas que se utiliza por vía tópica, actúa bloqueando la síntesis de prostaglandinas, estabiliza las membranas y previene las lesiones vasculares ${ }^{25-27}$.

El ácido hialurónico es un polímero natural presente en la dermis en condiciones normales y que actúa en los procesos 
de cicatrización mediante la estimulación de granulocitos y fibrina y la proliferación de fibroblastos ${ }^{28}$. Del mismo modo, el alginato sódico, un polímero de cadena larga que se extrae de las algas marinas, se ha empleado frecuentemente para el tratamiento de heridas exudativas y pude tener utilidad en el seno de epitelitis agudas de moderada-severa intensidad ${ }^{29}$.

El empleo de corticoesteroides para el tratamiento de la epitelitis se basa en sus conocidas propiedades antiinflamatorias. Si bien se han utilizado ampliamente en radioterapia, su validez continua siendo controvertida, ya que varios estudios no demostraron beneficio en los pacientes tratados con corticoesteroides tópicos. Una posible explicación estaría en la utilización de corticoides de baja potencia, en la heterogeneidad de los pacientes y tratamientos administrados en estos estudios y en la enorme variabilidad en su administración. Recientes ensayos utilizando corticoesteroides tópicos de mediana-alta potencia sí que han mostrado beneficios significativos en el tratamiento de la radiodermitis aguda ${ }^{30}$.

Otros fármacos y sustancias empleados tanto en el tratamiento como en la prevención de la toxicidad aguda sobre piel y mucosas han sido: misoprostol, análogo de la prostaglandina El que estimula la proliferación celular y la producción de moco ${ }^{31,32}$; azelastina, antihistamínico utilizado habitualmente en el tratamiento de procesos alérgicos, posee propiedades antiinflamatorias; glutamina, aminoácido no esencial con reconocido efecto protector sobre el tracto gastrointestinal durante la radioterapia ${ }^{33}$; hidrogeles e hidrocoloides, apósitos utilizados en enfermos con quemaduras severas, estarían indicados en el tratamiento de la descamación húmeda porque favorecen la cicatrización y reducen el tiempo necesario para la misma ${ }^{3,34}$.

\section{Inmunomoduladores y estimuladores de la regeneración} celular

El acúmulo local de neutrófilos derivado de la administración sistémica de los factores estimulantes de colonias de granulocitos (G-CSF) o de granulocitos-macrófagos (GMCSF) en pacientes sometidos a quimioterapia intensiva $y / 0$ trasplante de médula ósea ha demostrado aumentar los mecanismos de defensa de la mucosa oral y permitir una cicatrización más rápida de la mucosa. Se han observado también resultados prometedores al utilizar ambos factores por vía tópica para la cicatrización de heridas y úlceras varicosas crónicas. Esta evidencia ha llevado a intentar evaluar su efecto tanto en utilización sistémica como tópica en enjuagues en la prevención y el tratamiento de la mucositis oral radioinducida 35,36 .

La utilización de láseres de baja energía de $\mathrm{He} / \mathrm{Ne}$ ha demostrado que acelera la reepitelización, pudiendo por ello tener utilidad en la prevención y tratamiento de la mucositis ${ }^{37}$.

\section{Radioprotectores: amifostina, orgoteína}

Aunque la indicación aprobada de este fármaco es la prevención de la xerostomía a largo plazo tras radioterapia, numerosos ensayos han incidido en su posible papel en la prevención y atenuación de los síntomas de la mucositis aguda radioinducida tanto en pacientes con cáncer colorrectal o de cabeza y cuello. En 1999 la Sociedad Americana de On- cología Clínica (ASCO) recomendó la utilización de amifostina para disminuir la incidencia y severidad de la mucositis aguda durante la radioterapia así como de la toxicidad tardía en pacientes con tumores de cabeza y cuello tratados con radioterapia ${ }^{38-44}$.

La orgoteína (superóxido simutasa (SOD)), es una metaloproteína con capacidad para catalizar el radical superóxido a peróxido de hidrógeno y oxígeno impidiendo su acción sobre las membranas celulares y los ácidos nucleicos. Además, posee propiedades antiinflamatorias, antivirales y quimiotácticas.

La orgoteína es capaz de controlar los efectos secundarios de la radioterapia sin disminuir la eficacia antitumoral. Diversos ensayos han demostrado su eficacia tanto en el tratamiento como en la prevención de la toxicidad tardía, principalmente fibrosis subcutánea, como aguda, especialmente epitelitis, mucositis, enteritis o cistitis ${ }^{45-49}$.

\section{Conclusiones}

La mucositis aguda es un fenómeno inherente a la radioterapia en los tratamientos de tumores de cabeza y cuello. Pese a la gran cantidad de medidas y fármacos empleados sólo unos pocos han demostrado su utilidad en ensayos aleatorizados. Nuevos fármacos analgésicos como la capsaicina, inmunomoduladores como el AM3 o la administración tópica de inmunoglobulinas se perfilan como nuevas formas de tratamiento de esta complicación.

El manejo actual no deja de ser paliativo, encaminado principalmente al control de los síntomas hasta la reparación fisiológica del epitelio de la mucosa. Sin embargo, emerge con fuerza el concepto de prevención frente a tratamiento, sobre todo en lo que a las complicaciones tardías del tratamiento se refiere, empleando fármacos con actividad reconocida en la prevención pero que no disminuyan la eficacia del tratamiento.

\section{Bibliografía}

1. Sutherland SE, Browman GP. Prophylaxis of oral mucositis in irradiated head and neck cancer patients: a proposed classification scheme of interventions and meta-analysis of randomized controlled trials. Int J Radiat Oncol Biol Phys 2001; 49:917-30.

2. Barkham A. Radiotherapy skin reactions. Professional Nurse 1993; 8:732-6.

3. Pickering DG, Warland $\mathrm{S}$. The management of desquamative radiation skin reactions. Dressing Times 1992; 5.

4. Cox JD, Stetz J, Pajak TF. Toxicity criteria of the Radiation Therapy Oncology Group (RTOG) and the European Organization for Research and Treatment of Cancer (EORTC) Int J Radiat Oncol Biol Phys 1995; 30 (31):1341-6.

5. Shieh SH, Wang ST, Tsai ST, Tseng CC. Mouth care for nasopharyngeal cancer patients undergoing radiotherapy. Oral Oncol 1997; 33:36-41.

6. Campbell IR, Illingworth MH. Can patients wash during radiotherapyto the breast or chest wall? A randomized controlled trial. Clin Oncol (R Coll Radiol) 1992; 4:78-82.

7. Roy I, Fortin A, Larochelle M. The impact of skin washing with 


\section{A. Montero Luis y cols.}

water and soap during breast irradiation: a randomized study. Radiother Oncol 2001; 58:333-9.

8. Adamietz IA, Rahn R, Bottcher HD, et al. Prophylaxis with povidone-iodine against induction of oral mucositis by radiochemotherapy (see comments). Support Care Cancer 1998; 6:373-7.

9. Foote RL, Loprinzi CL, Frank AR, et al. Randomized trial of a chlorhexidine mouthwash for alleviation of radiation-induced mucositis. J Clin Oncol 1994; 12:2630-3.

10. Okuno $\mathrm{SH}$, Foote RL, Loprinzi $\mathrm{CL}$, et al. A randomized trial of a nonabsorbable antibiotic mucositis. Cancer 1997; 79:2193-9.

11. Rahn R, Adamietz IA, Boettcher HD, et al. Povidone-iodine to prevent mucositis in patients during antineoplastic radiochemotherapy. Dermatology 1997; 195:57-61.

12. Fidler $P$, Loprinzi $C L, O^{\prime} F a l l o n ~ J R$, et al. Prospective evaluation of a chamomile mouthwash for prevention of 5-FU-induced oral mucositis. Cancer 1996; 77:522-5.

13. Fenig $E$, Brenner B, Katz A, et al. Topical Biafine and Lipiderm for the prevention of radiation dermatitis: a randomized prospective trial. Oncol Rep 2001; 8:305-9.

14. Fisher J, Scott C, Stevens R, et al. Randomized phase III study comparing Best Supportive Care to Biafine as a prophylactic agent for radiation-induced skin toxicity for women undergoing breast irradiation: Radiation Therapy Oncology Group (RTOG) 97-13. Int J Radiat Oncol Biol Phys. 2000 1; 48:1307-10.

15. Halperin EC, Gasper L, George $S$ et al. A double blind randomized, prospective trial to evaluate topical vitamin $C$ solution for the prevention of radiation dermatitis. Int J Radiat Oncol Biol Phys 1993; 26:413-6.

16. Heggie S, Bryant GP, Tripcony L, et al. A Phase III study on the efficacy of topical aloe Vera gel on irradiated breast tissue. Cancer Nurs 2002; 25:442-51.

17. Iglesias A, Marín A, Barragán JA et al. Estudio sobre la respuesta dérmica en pacientes tratados con aloe vera en tratamientos radioterápicos. Rev Int Drmato Dermocos Clin SANED 2002; 5:466.

18. Lokkevik E, Skovlund E, Reitan JB, Hannisdal E, Tanum G. Skin treatment with bepanthen cream versus no cream during radiotherapy-a randomized controlled trial. Acta Oncol 1996; 35:1021-6.

19. Cerezo L, Iglesias $A$, Chinchetru $C$ et al. Efectos del lavado e hidratación de la piel durante el tratamiento radioterápico. Resultados de un estudio randomizado. Rev Oncología 2001; 3 Sup 1:183.

20. Carter DL, Hebert ME, Smink K, et al. Double-blind randomized trial of sucralfate vs placebo during radical radiotherapy for head and neck cancers. Head Neck 1999; 21:760-6.

21. Cenzig M, Ozyar E, Ozturk D, et al. Sucralfate in the preventionof radiation induced oral mucositis. J Clin Gastroenterol 1999; 28:40-3.

22. Makkonen TA, Bostrom P, Vilja $P$, Joensuu $H$. Sucralfate mouth washing in the prevention of radiation-induced mucositis: a placebo-controlled double-blind randomized study. Int J Radiat Oncol Biol Phys 1994; 30:177-82.

23. Maiche A, Isokangas OP, Grohn P. Skin protection by sucralfate cream during electron beam therapy. Acta Oncol 1994; 33:201-3.

24. Barker G, Loffus L, Cuddy P, Barker B.The effects of sucralfate suspension and diphenhydramine syrup plus kaolin-pectin on radiotherapy-induced mucositis. Oral Surg Oral Med Oral Pathol 1991; 71:288-93.

25. Epstein JB, Stevenson-Moore P, Jackson S, et al. Prevention of oral mucositis in radiation therapy: a controlled study with benzydamine hydrochloride rinse. Int J Radiat Oncol Biol Phys $1989 ; 16: 1571-5$.

26. Kim JH, Chu FC, Lakshmi V, Houde R. Benzydamine $\mathrm{HCl}$, a new agent for the treatment of radiation mucositis of the oropharynx. Am J Clin Oncol 1986; 9:132-4.

27. Samaranayake LP, Robertson AG, MacFarlane TW, et al. The effect of chlorhexidine and benzydamine mouthwashes on mucositis induced by therapeutic irradiation. Clin Radiol 1988; 39:291-4

28. Liguori V, Guillemin C, Pesce GF, et al. Double-blind, randomized clinical study comparing hyaluronic acid cream to placebo in patients treated with radiotherapy Radiother Oncol 1997; 42:155-61.

29. Oshitani T, Okada K, Kushima T, et al. [Clinical evaluation of sodium alginate on oral mucositis associated with radiotherapy]. Nippon Gan Chiryo Gakkai Shi 1990; 25:1129-37.

30. Boström A, Lindman $H$, Swartling $C$ et al. Potent corticosteroid cream (mometasone furoate) significantly reduces acute radiation dermatitis: results from a double-blind, randomized study. Radiother Oncol 2001; 59:257-65.

31. Porteder H, Rausch E, Kment G,Watzek G, Matejka M, Sinzinger $\mathrm{H}$. Local prostaglandin E2 in patients with oral malignancies undergoing chemo- and radiotherapy. J Craniomaxillofac Surg 1988; 16:371-4.

32. Hanson WR, Marks JE, Reddy SP. Protection from radiation-induced oral mucositis by a mouth rinse containing the prostaglandin El analog Misoprostol: a placebo controlled double blind clinical trial. Honn K, Ed. New York: Plenum Press; 1997.

33. Osaki T, Ueta E, Yoneda K, et al. T. Prophylaxis of oral mucositis associated with chemoradiotherapy for oral carcinoma by Azelastine hydrochloride (Azelastine) with other antioxidants. Head Neck 1994; 16:331-9.

34. Marks J. Mucosal protectants and their application for head and neck chemoirradiation. Curr Opin Oncol 1997; 2: 26773.

35. Saarilahti K, Kajanti M, Joensuu $T$, et al. Comparison of granulocyte-macrophage colony-stimulating factor and sucralfate mouthwashes in the prevention of radiation-induced mucositis: a double-blind prospective randomized phase III study. Int J Radiat Oncol Biol Phys 2002; 54(2):479-85.

36. Schneider SB, Nishimura RD, ZimmermanRP, et al. Filgrastim ( $r$-metHuG-CSF) and its potential use in the reduction of radiationinducedoropharyngeal mucosiis: an interimlook at a randomized, double-blind, placebocontrolledtrial. Cytokines Cell Mol Ther 1999; 5:175-80.

37. Bensadoun RJ, Franquin JC, Ciais $G$, et al. Low-energy $\mathrm{He} / \mathrm{Ne}$ laser in the prevention of radiation-induced mucositis.A multicenter phase III randomized study in patients with head and neck cancer (see comments). Support Care Cancer 1999; 7:244-52

38. Antonadou D, Pepelassi M, Synodinou M, et al. Prophylactic use of amifostine to prevent radiochemotherapy-induced mucositis and xerostomia in head-and-neck cancer. Int J Radiat Oncol Biol Phys 2002; 52(3):739-47

39. Bourhis J, De Crevoisier R, Abdulkarim B, et al. A randomized study of very accelerated radiotherapy with and without amifostine in head and neck squamous cell carcinoma. Int J Radiat Oncol Biol Phys 2000; 46:1 105-8.

40. Brizel DM, Wasserman TH, Henke $M$, et al. Phase III randomized trial of amifostine as a radioprotector in head and neck cancer. J Clin Oncol 2000; 18(19):3339-45. 
41. Buntzel J, Kuttner K, Frohlich D, Glatzel M. Selective cytoprotection with amifostine in concurrent radiochemotherapy for head and neck cancer (see comments). Ann Oncol 1998; 9:505-9.

42. Koukourakis MI,Kyrias G, Kakolyris S, et al. Subcutaneous administration of amifostine during fractionated radiotherapy: a randomized phase II study. J Clin Oncol 2000; 18:2226-33.

43. Vacha P, Marx M, Engel A, et al. [Side effects of postoperative radiochemotherapy with amifostine versus radiochemotherapy alone in head and neck tumors. Preliminary results of a prospective randomized trial.]Strahlenther Onkol 1999; 175 Suppl 4:18-22.

44. Hensley ML, Schuchter LM, Lindley C, et al. American Society of Clinical Oncology clinical practice guidelines for the use of chemotherapy and radiotherapy protectants. J Clin Oncol 1999 17:3333-55.

45. Valencia J, Velilla C, Urpegui A, et al.Bascon N, Esco R. The ef- ficacy of orgotein in the treatment of acute toxicity due to radiotherapy on head and neck tumors. Tumori. 2002 Sep-Oct; 88(5):385-9.

46. Lefaix JL, Delanian S, Leplat JJ, et al. [Radiation-induced cutaneo-muscular fibrosis (III): major therapeutic efficacy of liposomal Cu/Zn superoxide dismutase] Bull Cancer 1993 Sep; 80(9):799-807.

47. Delanian $S$, Martin $M$, Housset $M$. [latrogenic fibrosis in cancerology (1): descriptive and physiopathological aspects] Bull Cancer 1993 Mar; 80(3):192-201.

48. Delanian S, Lefaix JL, Housset M. [latrogenic fibrosis in cancerology (2): main etiologies and therapeutic possibilities] Bull Cancer 1993 Mar; 80(3):202-12.

49. Ruiz Martín V, Algara López M, Foro Arnalot A, et al. Eficacia de la orgoteina en el tratamiento de la fibrosis y xerostomía radioinducidas. Oncología 1995; 18:60-4. 